\title{
2019 SNOOK Prizes in Computational Statistical Mechanics
}

\author{
Wm.G. Hoover, C.G. Hoover \\ Ruby Valley Research Institute \\ Highway Contract 60, Box 601, Ruby Valley, Nevada 89833, USA \\ E-mail: hooverwilliam@yahoo.com
}

Published online: 14 March 2019

\begin{abstract}
The one-dimensional $\phi^{4}$ Model generalizes a harmonic chain with nearest-neighbor Hooke's-Law interactions by adding quartic potentials tethering each particle to its lattice site. In their studies of this model Kenichiro Aoki and Dimitri Kusnezov emphasized its most interesting feature: because the quartic tethers act to scatter long-wavelength phonons, $\phi^{4}$ chains exhibit Fourier heat conduction. In his recent Snook-Prize work Aoki also showed that the model can exhibit chaos on the threedimensional energy surface describing a two-body two-spring chain. That surface can include at least two distinct chaotic seas. Aoki pointed out that the model typically exhibits different kinetic temperatures for the two bodies. Evidently few-body $\phi^{4}$ problems merit more investigation. Accordingly, the 2019 Prizes honoring Ian Snook (1945-2013) [five hundred United States dollars cash from the Hoovers and an additional \$500 cash from the Institute of Bioorganic Chemistry of the Polish Academy of Sciences and the Poznan Supercomputing and Networking Center] will be awarded to the author(s) of the most interesting work analyzing and discussing few-body $\phi^{4}$ models from the standpoints of dynamical systems theory and macroscopic thermodynamics, taking into account the model's ability to maintain a steady-state kinetic temperature gradient as well as at least two coexisting chaotic seas in the presence of deterministic chaos.
\end{abstract}

Details of the Prize Problem can be found on pages 159-162 of Volume 24(2) of Computational Methods in Science and Technology (CMST) http://dx.doi.org/10.12921/cmst.2018.0000032. No Prize was awarded in 2018.

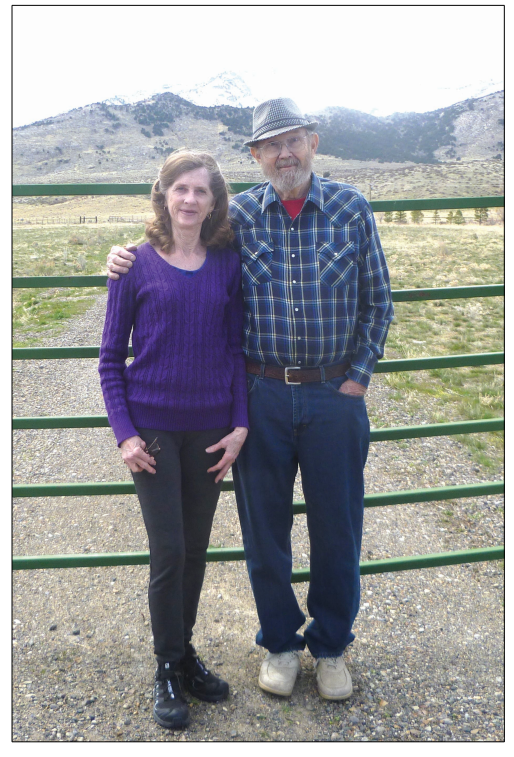

William G. Hoover, Carol G. Hoover; Bill and Carol Hoover live in Ruby Valley (Elko County Nevada) where the cows outnumber the people by orders of magnitude and the phonebook is a single page. Their research interests include chaos, fractals, symmetry breaking instabilities, and computer simulations linking the microscopic and macroscopic descriptions of nonequilibrium processes. Their most recent book is Microscopic and Macroscopic Simulation Techniques - Kharagpur Lectures (World Scientific Publishers, Singapore, 2018). 